Impacto de las redes sociales como un nuevo canal de comercialización para microempresarios de Gran Asunción. Periodo 2004-2014

\title{
The impact of social networks as a new path of commercialization for micro entrepreneurs in Gran Asunción, Period 2004-2014
}

\author{
Claudia Emilia Torres Zárate $\left(^{*}\right)$ \\ $\left.{ }^{*}\right)$ Graduado en Economía. Facultad de Ciencias Económicas, \\ San Lorenzo- Paraguay \\ Correo electrónico: claudita.torres@hotmail.com \\ Tutor: Econ. Víctor Ruíz Díaz. \\ Recibido:08/10/15; Aceptado: 10/11/15
}

\section{Resumen}

Este artículo tiene por objeto conocer el impacto económico y social de las redes sociales en la zona de Gran Asunción', como una nueva modalidad estratégica de negocios en el campo de los microempresarios, verificado a través de la penetración de internet en Paraguay, durante la década 20042014.

Se describe la red social Facebook, líder del país para los negocios en la actualidad; seguidamente se presenta el ránking de las redes sociales con mayor tráfico en el país, y su importancia para los microempresarios y consumidores. Se incluye además uno de los casos de éxito en el Paraguay.

Por último, se desarrolla la representación y el análisis de los resultados estadísticos obtenidos a partir de las encuestas practicadas a los usuarios de internet. Por lo tanto, a través de los estudios realizados se logra demostrar la influencia positiva de las redes sociales para el comercio de los microempresarios en el área metropolitana de Asunción.

Palabras clave: impacto, redes, microempresarios
Abstract

This study aims to discover the social economic impact in the Gran Asuncion Area, as a new business strategy in the sector of micro entrepreneurs, verified through the incorporation of the Internet in Paraguay, during the $2004-2014$ decade.

The social network Facebook is described, as the leading network for businesses at the present time; sequentially the ranking of the most frequented social networks, and its importance to micro entrepreneurs and consumers is presented. One of the cases with greater success in Paraguay is included as well. Lastly, the presentation and analysis of the results obtained through the surveys pursued by Internet users is developed. Therefore, through the studies conducted, the positive influence of social networks for the market of micro entrepreneurs of Asuncion metropolitan area can be demonstrated.

Key words: impact, networks, microentrepreneurs

${ }^{1}$ Gran Asunción: equivalente al área metropolitana de Asunción 


\section{Introducción}

Este trabajo de investigación pretende presentar y discutir las oportunidades que ofrecen las redes sociales para fortalecer $y$ acrecentar los negocios de las microempresas, que buscan mejorar su bienestar económico y social, mediante la participación en nuevos mercados, a partir de este nuevo modelo de negociación.

Este mecanismo contribuye además en un mayor crecimiento socioeconómico del país, pues el sector representa más del $90 \%$ del total de las empresas legalmente constituidas en el Paraguay, según los datos recopilados por el Censo Económico Nacional llevado a cabo en el año 2012.

Con la aparición de este nuevo fenómeno, se abren nuevos canales de comercialización moderna, por lo que la investigadora centra su atención en la importancia de las redes sociales como instrumento captador de mayores beneficios en emprendimientos de carácter micro.

De ahí surge el problema científico, el cual es: ¿Qué beneficios socioeconómicos obtiene el microempresario de Gran Asunción a través del comercio en las redes sociales?, por lo que se estableció el objetivo de determinar esos beneficios.

\section{Materiales y Métodos}

Para la medición de la transcendente evolución de Internet en el país se tomó como periodo de estudio a partir del año 2004 al 2014, así como el análisis de la utilidad de las redes sociales para el año 2015.

Por lo tanto se propuso la siguiente hipótesis: el comercio en las redes sociales ha afectado positivamente en los beneficios socioeconómicos de los microempresarios de Gran Asunción, cuya variable dependiente se refiere a los beneficios socioeconómicos de los microempresarios en Gran Asunción, mientras que la variable independiente es el comercio en las redes sociales.

Para esa finalidad se recurre a la recolección de datos que comprenden fuentes primarias (encuesta electrónica a microempresarios y consumidores) y secundarias como documentos de la Comisión Nacional de Telecomunicaciones (Conatel), de la Dirección Nacional de Estadística, Encuestas y Censos (DGEEC), libros de textos y consultas de artículos de Internet, blogs, periódicos digitales, revistas, entre otros.

Las encuestas estuvieron habilitadas en las redes sociales del 15 de marzo al 15 de abril del 2015, bajo las siguientes denominaciones: "Uso de las redes sociales como medio comercial" y "Beneficio de las redes sociales", en los enlaces http://goo.gl/ forms/o5dSsGafeA y http://goo.gl/forms/ p8PGX7dcWg, respectivamente. El primero estaba constituido por 19 preguntas cerradas; mientras que el segundo, por un total de cuatro. En ambos casos, la muestra abarcó 230 personas.

El cuestionario se estructuró según los elementos o aspectos de relevancia social y económica, tales como: porcentaje de microempresarios que denotan beneficios a través de las redes sociales para sus negocios, proporción de los usuarios que ya han utilizado este medio para realizar compras, además de cuáles son las redes con mayor incidencia en el sector comercial.

Estos parámetros fueron procesados en microsoft excel para obtener la información estadística que demuestre la efectividad de la utilización de estas redes como estrategia para los microempresarios respecto a sus emprendimientos. 


\section{Resultados y Discusión}

A medida que el tiempo avanza, mayor es el número de usuarios que se van integrando a las distintas redes sociales existentes en Internet. Frente a esta tendencia en creciente aumento, los microempresarios detectaron un nuevo canal de comercialización que estaba siendo desaprovechado y que correctamente utilizado podía proveerles grandes beneficios socioeconómicos.

\section{Tabla 1. Penetración y crecimiento de Internet en Paraguay Década 2004-2014}

\begin{tabular}{|c|c|c|c|c|}
\hline Año & $\begin{array}{c}\text { \% Penetración (Po- } \\
\text { blación con internet) }\end{array}$ & $\begin{array}{c}\text { \%crecimiento de } \\
\text { usuarios interanual }\end{array}$ & $\begin{array}{c}\text { Población de } 10 \\
\text { años y más de edad }\end{array}$ & $\begin{array}{c}\text { Usuarios total de } \\
\text { Internet }\end{array}$ \\
\hline 2004 & $3,2 \%$ & $18,8 \%$ & 4.354 .918 & 142.542 \\
\hline 2005 & $4,4 \%$ & $40,3 \%$ & 4.498 .263 & 200.000 \\
\hline 2006 & $8 \%$ & $83,5 \%$ & 4.609 .121 & 366.982 \\
\hline 2007 & $11,2 \%$ & $44,66 \%$ & 4.734 .593 & 530.876 \\
\hline 2008 & $15,1 \%$ & $37,55 \%$ & 4.840 .821 & 730.196 \\
\hline 2009 & $18,9 \%$ & $29,9 \%$ & 5.028 .575 & 948.620 \\
\hline 2010 & $19,8 \%$ & $6,54 \%$ & 5.111 .009 & 1.010 .676 \\
\hline 2011 & $24,8 \%$ & $29,8 \%$ & 5.297 .713 & 1.311 .900 \\
\hline 2012 & $29,2 \%$ & $20,25 \%$ & 5.309 .235 & 1.577 .591 \\
\hline 2013 & $36,9 \%$ & $28,74 \%$ & 5.502 .504 & 2.030 .925 \\
\hline 2014 & $44 \%$ & $21,8 \%$ & 5.617 .211 & 2.473 .724 \\
\hline
\end{tabular}

Fuente: Elaboración propia en base a los informes anuales de la Encuesta Permanente de Hogares, la Comisión Nacional de Telecomunicaciones y la UIT ( Unión Internacional de Telecomunicaciones).

Analizando los datos de la década 20042014, en la tabla 1 se destaca el potencial crecimiento del acceso a internet pasando de miles a millones de usuarios, en términos porcentuales en el año 2004 la penetración fue del $3,2 \%$ y el crecimiento interanual del $18,8 \%$ respecto al año anterior cuya suma alcanzaba el total de 120.000 de la PEA². Al año siguiente, la penetración aumentó 1,2 puntos porcentuales, quedando en $4,4 \%$ y un crecimiento de usuarios de $21,5 \%$ alcanzando un $40,3 \%$ en el 2005.
Posteriormente, en el año 2008, con la liberación del acceso a internet, el cual finalizó con el monopolio por parte de Copaco, se empezó a obtener un mayor alcance y ya para el 2014 llegó a un total de 2.473.724 usuarios equivalente a una penetración del $44 \%$. De esta manera se evidencia un crecimiento exponencial de $1.635 \%$ aproximadamente, con respecto al año base 2004, representado en la figura 1.

${ }^{1}$ Población económicamente activa (población de 10 años y más de edad). 


\section{Figura 1. Crecimiento porcentual de usuarios de Internet, Paraguay 2004-2014}

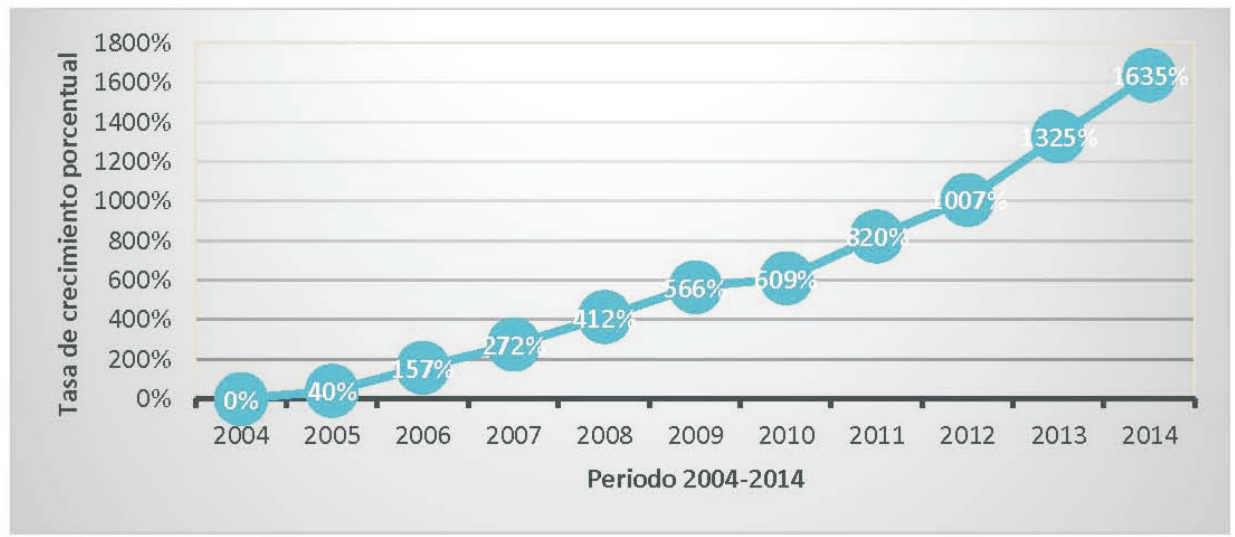

Fuente: Elaboración propia en base a los informes anuales de la Encuesta Permanente de Hogares, la Comisión Nacional de Telecomunicaciones y la UIT ( Unión Internacional de Telecomunicaciones)

El Paraguay cuenta con una cifra total de 1.802.088 dispositivos móviles con acceso a internet y redes sociales, se denotó un crecimiento del 56\% entre febrero del 2014 y febrero del 2015 en los teléfonos inteligentes con acceso a las redes (Conatel, 2015).

Por otro lado, Asunción supera la media de conectividad del país con una cifra que alcanza 293.605 usuarios de internet, lo que corresponde a una penetración del $65,8 \%$ de la PEA. A nivel de Gran Asunción, las principales ciudades con mayor acceso son Luque y San Lorenzo con un $36,4 \%$ de participación entre ambas.

La red es una herramienta vital para la economía, la población mundial se acostumbra cada vez más a comerciar a través de ella, fundamentalmente por medio de los dispositivos móviles (García, J., 2013).

La Red Social líder del país para negocios. Facebook. Caso de éxito en Paraguay.

En marzo del 2015 se dio a conocer la cantidad de perfiles paraguayos en Facebook que alcanzó un total de 2.600 .000 usuarios, ocupando de esa manera el puesto 66 en el ranking mundial. Teniendo en cuenta la población de 10 años y más de edad, la penetración de esta red social asciende a $46,3 \%$, casi la mitad de la población nacional (Agencia digital Latamclick, 2015).

Figura 2. Usuarios de Facebook en Paraguay Periodo 2011-2015

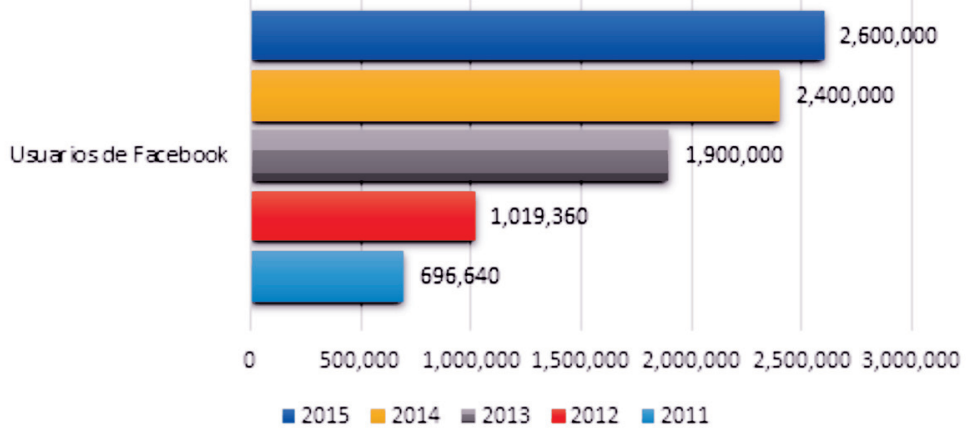

Fuente: Elaboración propia en base a datos de Agencia Digital Latamclick y Socialbakers. 
En la figura 2 se aprecia que en el año 2011 el número de usuarios paraguayos de Facebook alcanzaba un total de 696.640, para el siguiente año la cifra ascendía a 1.019.360, mientras que en el 2013 ya se contaba con 1.900.000, lo que implicó un crecimiento de $173 \%$ respecto al 2011. En el año 2014 la cifra de usuarios alcanzó los 2.400.000, y para el 2015 ya se cuenta con un total de 2.600 .000 usuarios de Facebook en nuestro país, y considerando el año base (2011), el crecimiento es de $273 \%$.

En mayo del 2015, Facebook contaba con 40 millones de Fanpages de pequeños y medianos negocios, para los cuales anunciaron Facebook Fit, un programa específico para este tipo de microempresas, con el que se pretende que las mismas obtengan los mejores resultados económicos (Agencia Digital Latamclick, 2015).

Existen varios casos de éxito en el país, como el caso de "Hendyla", la primera red social de ventas en el país. Esta comunidad ya cuenta con más de 230 mil usuarios en el 2015, que efectúan cerca de 400 transacciones diarias (Diario 5días, 2015).

\section{La efectividad de las Redes Sociales como canal de comercialización para microempresarios de Gran Asunción}

Esta nueva modalidad de negocio afecta positivamente tanto a oferentes como a consumidores, puesto que permite servicios mucho más eficientes tales como: crear una comunidad de la microempresa, realizar análisis de mercado demográficos, visualizar los productos y/o servicios que se ofrecen las 24 horas del día, realizar pedidos sin necesidad de salir del lugar en donde uno se encuentre, tomar decisiones de compras adquiriendo información de manera inmediata, entre otras.

La encuesta que se creó a través de Internet concluyó que el total de los encuestados cuenta con un perfil en alguna red social, el $99 \%$ de los mismos ya acceden desde sus dispositivos móviles. Además, el $63 \%$ de los mismos indicó que el $100 \%$ de sus amigos tiene presencia en las redes y el $92 \%$ tiene contactos que las utilizan como canal de negocios, siendo Facebook el que percibe más anuncios (96,5\%), seguido de Instagram (86,5\%).

En cuanto al género de los encuestados, el $42 \%$ corresponde al sexo masculino, en tanto que el $58 \%$ restante al femenino. con respecto a la franja etaria de la muestra, el $70 \%$ pertenece al rango de 18-25 años de edad, el 23\% entre 26-35 años y el 7\% a los de 35 años y más. Por otro lado, también se destacó que el mayor tiempo promedio que un usuario dedica a una red social es de 2 a 5 horas, es decir, equivalente al $52 \%$ de los consultados; mientras que el $26 \%$ le dedica una hora y el otro $22 \%$ más de 5 horas.

Las redes sociales de mayor tráfico son Facebook (36\%), Instagram (33\%), seguidos en menor porcentaje por YouTube (14\%), Snapchat (10\%) y Twitter (7\%).

\section{Figura 3. Bienes y servicios ofrecidos en la red social, Área Metropolitana de Asunción - Paraguay, 2015}

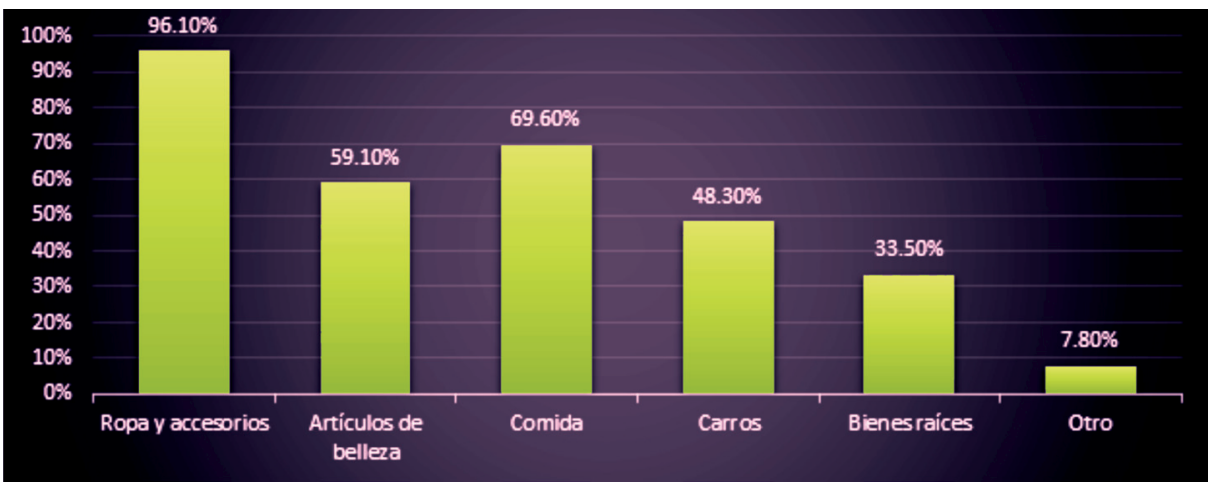

Fuente: Elaboración propia en base a datos del estudio estadístico. 
En la figura 3 se denota que el bien ofrecido con mayor frecuencia corresponde a las ropas y accesorios (96\%); seguido de alimentos
(70\%), artículos de belleza (59\%), carros (48\%), bienes de raíces (34\%) y otros $(8 \%)$.

Figura 4. Compras por redes sociales, Área Metropolitana de Asunción - Paraguay, 2015

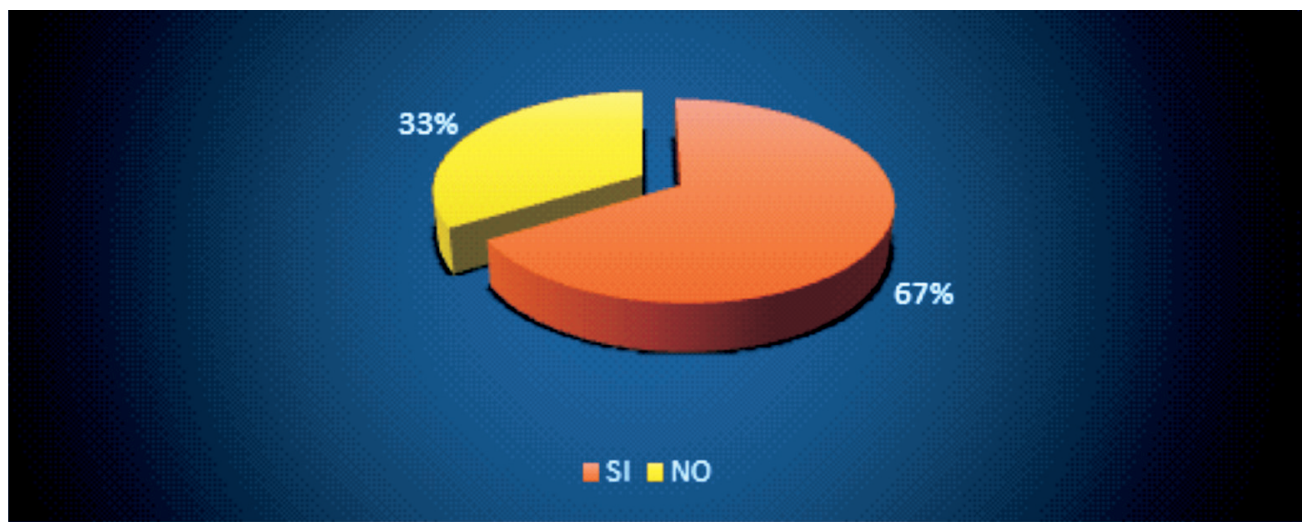

Fuente: Elaboración propia en base a datos del estudio estadístico.

En la figura 4, se observa que el $67 \%$ de los encuestados realizó más de una vez alguna transacción, mientras que el 33\% aún no lo ha hecho. Un importante resultado, puesto que supera el $50 \%$, equivalente a los tres cuartos de los encuestados.

\section{Figura 5. Beneficio de uso comercial de la red social, Área Metropolitana de Asunción - Paraguay, 2015}

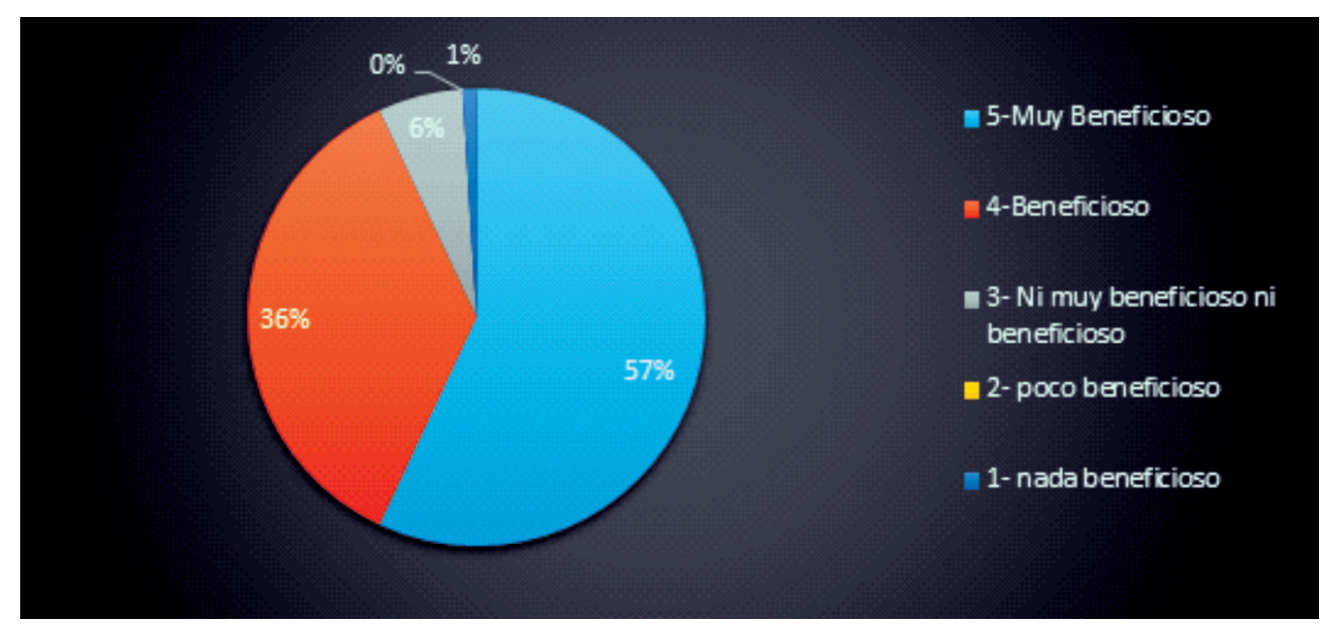

Fuente: Elaboración propia en base a datos del estudio estadístico.

Los datos estadísticos del estudio indicaron que el $57 \%$ de los microempresarios encuestados consideró a las redes sociales como "Muy Beneficiosa”, el 36\% sostienen que es "Beneficioso". Por otro lado, en un menor porcentaje, apenas el $6 \%$ de ellos, lo considera como "Ni muy beneficioso ni beneficioso" y $1 \%$ nada Beneficioso. 


\section{Figura 6. Disposición de local físico, Área Metropolitana de Asunción - Paraguay, 2015}

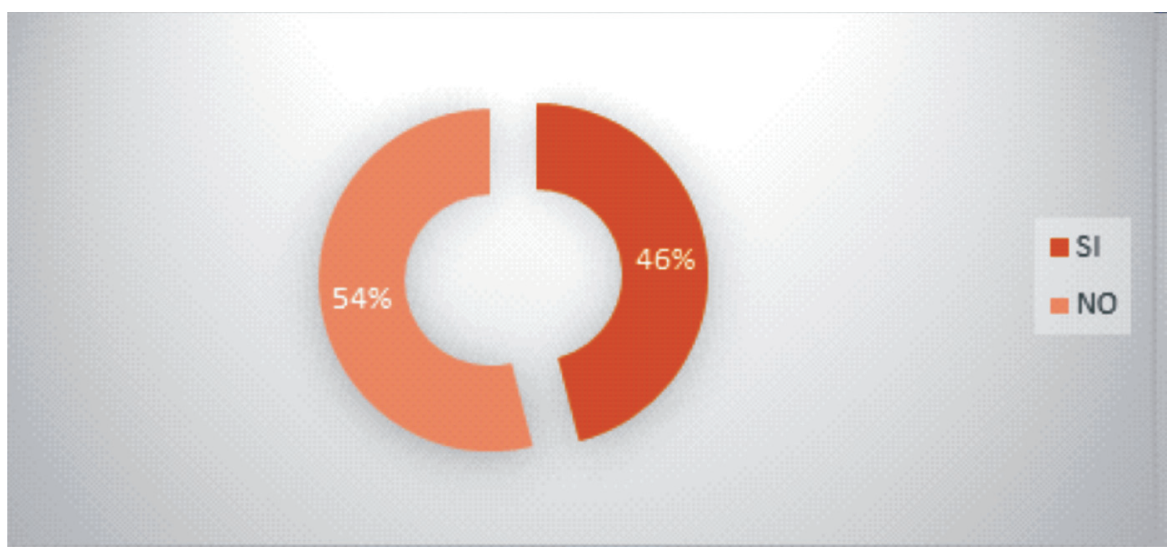

Fuente: Elaboración propia en base a datos del estudio estadístico.

Con relación a la figura 6 , se expone un resultado que indica que el $46 \%$ cuenta con un local, mientras que el $54 \%$ de los demás microempresarios aún no cuenta con un espacio físico.

Por lo tanto, de esta manera se corrobora el alto grado de satisfacción de los microempresarios de Gran Asunción sobre el uso de las redes sociales, que permite, inclusive a muchos con menor disponibilidad de capital, comenzar un negocio sin necesidad de contar desde un inicio con algún local comercial.

\section{Conclusiones}

Esta investigación resulta ser la primera que profundiza el impacto de las redes sociales sobre los micronegocios en el país, sin embargo su cuantificación es limitada, debido a que existen aún muy pocos datos respecto a las variables e indicadores alusivos al tema.

Se ha comprobado que la evolución del internet en el país ha sido exponencial considerando la década 2004-2014, siendo las redes sociales el principal motivo de estas conexiones en un $100 \%$. Sin duda, ésta es la era de la Internet, por lo que realizar acciones de negocios sin tener en cuenta este fenómeno es irracional.

En este momento no es posible que los emprendedores formen parte del mercado sin estar relacionados de alguna manera con los consumidores. Todos se hallan relacionados directa o indirectamente con las redes, convirtiéndola en el "boom" del momento.

Según los datos recopilados de la DGEEC y de la Conatel, en el periodo 2004-2014 el número de paraguayos con acceso a internet ha ido aumentando año tras año. En el 2004 apenas 142.542 personas tenían acceso y en el 2014 la cifra de conexiones aumentó a 2.473.724 de paraguayos, evidenciándose así un crecimiento considerable del $1.635 \%$ con respecto al año base 2004 .

El número de compatriotas registrados como usuarios de la red social Facebook, tanto residentes en el país como en el resto del mundo, fue de 2.600 .000 aproximadamente, para el año 2015 , equivalente a casi el $46,3 \%$ de la población económicamente activa.

Por lo tanto, este desarrollo tecnológico ha llevado a muchos emprendedores a desarrollar sus negocios y mejorar sus capacidades de transacción, dándole un alcance que permite traspasar las fronteras y lograr participar en el mercado con otras grandes y medianas empresas a costes bajos, transformándolo en el medio más efectivo, económico y eficaz para cualquier microempresa de la actualidad.

El gran incremento de esta nueva forma de hacer negocios se encuentra ligado al crecimiento de los dispositivos móviles con acceso a la red. Alrededor de 1.802.088 los dispositivos con acceso a la red hasta febrero 
del 2015 , de los cuales $69,3 \%$ son teléfonos inteligentes (Conatel, 2015).

Mediante este estudio se demostró que más del $67 \%$ de los encuestados ya realizó una compra a través de las redes sociales y, por su parte, más del $90 \%$ de los microempresarios expresó su satisfacción respecto a las mismas, debido a los beneficios obtenidos con ellas. Finalmente, por medio de la metodología utilizada en la investigación, se comprobó la existencia de grandes beneficios en el comercio de los microempresarios de Gran Asunción, derivados del uso de las redes sociales.

\section{Referencia Bibliográfica}

Conatel (2013). Matriz de Indicadores de Desarrollo de Telecomunicaciones 2010/2012. Paraguay.

Conatel (2014). Matriz de Indicadores de Desarrollo de Telecomunicaciones 2013. Paraguay.

Conatel (2015). Cantidad de usuarios por tecnología de acceso a Internet. Paraguay.

Conatel (2015). Matriz de Indicadores de Desarrollo de Telecomunicaciones 2014. Paraguay.

Conatel (2014). Cantidad de usuarios por tecnología de acceso a Internet. Paraguay.

Diario 5 días. (18 de abril de 2015). Obtenido de www.diario5días.com

DGEEC (2005). Encuesta Permanente de Hogares 2004. Total País.

DGEEC (2006). Encuesta Permanente de Hogares 2005. Total País.

DGEEC (2007). Encuesta Permanente de Hogares 2006. Total País.

DGEEC (2008). Encuesta Permanente de Hogares 2007. Total País.

DGEEC (2009). Encuesta Permanente de Hogares 2008. Total País.

DGEEC (2010). Encuesta Permanente de Hogares 2009. Total País.

DGEEC (2011). Encuesta Permanente de Hogares 2010. Total País.

DGEEC (2012). Encuesta Permanente de Hogares 2011. Total País.

DGEEC (2013). Encuesta Permanente de Hogares 2012. Total País.

DGEEC (2014). Encuesta Permanente de Hogares 2013. Total País.

DGEEC (2015). Encuesta Permanente de Hogares 2014. Asunción.

DGEEC (2015). Encuesta Permanente de Hogares 2014. Total País.

Abad, D. (27 de Mayo de 2015). Agencia de Social Media. Obtenido de www.latamclick.com

JanRezab, M. H. (3 de mayo de 2015). Empresa de Análisis de Medios Sociales. Obtenido de Socialbakers: www.socialbakers.com

Celaya, J. (2011). La Empresa en la Web 2.0: El impacto de las Redes Sociales y las nuevas formas de comunicación online es la estrategia empresarial. España: Illustrated.

Vaynerchuy, G. (2012). La economía de la gratitud: Traslada tus negocios a las redes sociales. México: Penguin RandomHouse Grupo Editorial 